\title{
Extraction of Solar Coronal Magnetic Loops with the Directional 2D Morlet Wavelet Transform
}

\author{
S. Biskri · J.-P. Antoine $\cdot$ B. Inhester $\cdot$ F. Mekideche
}

Received: 23 April 2009 / Accepted: 17 February 2010 / Published online: 6 March 2010

(C) The Author(s) 2010. This article is published with open access at Springerlink.com

\begin{abstract}
We present an automated extraction method based on the continuous wavelet transform (CWT) for analyzing solar magnetic loops. The aim of the work is to extract, from the images taken from solar EUV telescopes, the traces of bright loops presumably shaped by the magnetic field of the solar corona. The technique is that of wavelet analysis, using the two-dimensional Morlet wavelet, because of its efficiency in detecting oriented features, which allows us to follow closely the curvature of the loops. Next, we segment the wavelet modulus image and we threshold it, both globally and locally (i.e., adaptively), in order to eliminate the remaining noise. Altogether, our method performs well, it is robust and fast, and could be used as a standard tool for analyzing large data sets expected from missions like SDO.
\end{abstract}

Keywords Corona $\cdot$ Magnetic loops · Continuous wavelet transform · Extraction · Segmentation

Solar Image Processing and Analysis

Guest Editors: J. Ireland and C.A. Young

S. Biskri · F. Mekideche

Laboratoire de Physique Théorique, Faculté de Physique, Univ. des Sciences et de la Technologie Houari Boumediène (USTHB), BP 32 El-Alia, 16111 Bab-Ezzouar, Alger, Algeria

S. Biskri

e-mail: sambiskri@gmail.com

F. Mekideche

e-mail: fazia_mekideche@yahoo.com

S. Biskri · J.-P. Antoine ( $\varangle)$

Institut de Physique Théorique, Université catholique de Louvain, 1348 Louvain-la-Neuve, Belgium e-mail: jean-pierre.antoine@uclouvain.be

B. Inhester

Max-Planck Institute for Solar System Research, 37191 Katlenburg-Lindau, Germany

e-mail: binhest@mps.mpg.de 


\section{Introduction}

Solar coronal images in EUV wavelengths exhibit among other features loop-like objects, which are believed to be a manifestation of magnetic flux tubes filled with hot plasma dense enough to emit the observed EUV signature.

From field measurements on the solar surface, it can be estimated that the solar corona is almost entirely permeated by a magnetic field of the order of ten Gauss. In the lower part of the corona, the magnetic-field energy density exceeds the thermal and bulk kinetic energy density by orders of magnitude. In the lower corona, where most of the EUV loop emission originates, the ratio $\beta=$ thermal pressure $/ \mu_{0} B^{2}$ corresponds to $\beta \approx 10^{-3}-10^{-1}$ (see for instance Gary, 2001). Inevitably, knowledge of the field, both in strength and topology, is therefore a prerequisite to the understanding of dynamical processes in the corona. The topology of the coronal magnetic field, however, is difficult to determine from magnetic-field models which are constructed from the surface magnetograms (e.g. Wiegelmann, 2008). Coronal EUV observations could help to constrain this topology and determine to some extent the field-line connectivity on the solar surface.

For this reason, there is great interest in extracting the shape of the loops from EUV images, and in using them to constrain the shape of field lines in the corona or even reconstructing some of them from a stereoscopic pair of images (Feng et al., 2007; Aschwanden et al., 2008b).

In this paper, we propose an efficient scheme to detect and extract loop structures from EUV images and we present an application of our approach to a Transition Region and Coronal Explorer (TRACE) observation of an active region. Our scheme, called Wavelet Transform-based loop Extraction method (WTE), is based on a suitable mathematical tool (wavelet analysis) which can effectively extract information of interest buried in signals of various nature. Of course, we still face the usual vision problem, namely, our method analyzes the projection of the loops on the plane orthogonal to the line of sight, not the 3D loops themselves. In addition, wavelet analysis by itself is not sufficient for properly detecting the loops. Thus we perform, in a second stage, a segmentation of the wavelettransformed image in order to improve the discrimination between genuine loop pixels and noise. Our method proves to be robust, in the sense that it does not generate false loops where there are none and, due to the use of wavelets, it is less prone to perturbations and noise in the image than other methods.

Wavelets are applied in many domains of physics. For instance, there is a wide range of applications in signal and image processing (Antoine et al., 1993), in astrophysics (Antoine et al., 2002a; Delouille et al., 2005), in fluid dynamics (Farge et al., 1998), and in condensed matter physics. In astrophysics, the first attempt to apply the continuous wavelet transform (CWT) was performed by Slezak, Bijaoui, and Mars (1990). At present, wavelets have become common in cosmology, e.g. for analyzing the anisotropies of the Cosmic Microwave Background (CMB) (McEwen et al., 2006).

In this paper, we focus on segmentation and extraction of coronal loops in solar images, with help of the two-dimensional (2D) continuous wavelet transform (Antoine et al., 2004). Several methods for segmenting coronal-loop structures have been reported in the literature. The Oriented Connectivity-based Method (OCM) due to Lee, Newman, and Gary (2006b), which is based partly on Strous' loop pixel labeling (Strous, 2002), was the first automated coronal-loop-segmentation method proposed. The loop tracing in the OCM is heavily guided by the local magnetic-field direction estimated from simultaneous magnetograms. However, coronal EUV images often do not have corresponding magnetograms, so the OCM is not applicable in these cases. 
Another segmenting method, the Dynamic Aperture-based loop-segmentation Method (DAM), was introduced by Lee, Newman, and Gary (2006a). DAM segments coronal loops by searching for regions whose intensity profile is well characterized by a Gaussian shape. Indeed, Carcedo et al. (2003) reported that the coronal loops have a Gaussian-like distribution of their cross-sectional intensity profile. More recently, the Ridge detection by Automated Scaling (RAS) method, which is considered as an extension of Lee's OCM, has been developed by Inhester, Feng, and Wiegelmann (2008). The improvement consists in replacing Strous' ridge-point detection scheme by the modified multiscale approach of Lindeberg (1998), which automatically adjusts to a varying loop thickness and also returns an estimate of reliability of the ridge-point location and orientation. A detailed comparison of different algorithms for coronal-loop tracing has been reported by Aschwanden et al. (2008b).

In this sequel, we propose a new approach for extracting the bright loop features from solar EUV images. Our technique is based on processing EUV images with help of the 2D Morlet continuous wavelet transform (Torrésani, 1995; Mallat, 1999; Antoine et al., 2004). This wavelet efficiently detects the elongated structures from loops and their orientation in the image. Therefore additional information, e.g. from magnetogram data, is not needed. In this sense, our method is more general than OCM. We also make no assumptions on the intensity profile across the loop, contrary to DAM or RAS.

Clearly, blurred boundaries, close bundles and crossing of loops make the analysis quite complex. In addition, presumably EUV loop sections at larger heights often fade out and show a lower contrast above the background than the lower foot-point sections. All of these features make it desirable to use high-resolution images and deep exposures, and this is why we focus on TRACE images. The more so since the TRACE mission is primarily devoted to the investigation of coronal loops. We emphasize that the method itself does not require a minimum pixel size, since wavelets are freely rescalable. Therefore our method will in principle also work with EUV images of lower resolution than that of the TRACE images. However, the method is not capable of detecting loops not properly resolved in an image (e.g. subgrid structures, at least unless some a priori assumptions are made about them). Besides, whether a loop can be detected depends not only on its size relative to the image resolution, but also strongly on the image contrast: large, bright loops are discernable in lower-resolution images, smaller loops and loops bundles require higher resolution. In fact, it is not improbable that loops consist of a hierarchy of structures and threads below even the TRACE resolution (Aschwanden, Nightingale, and Alexander, 2000; Sakai and Furusawa, 2002). In any case, further applications of our WTE method should be made, for instance to STEREO/SECCHI images or other TRACE channels. We postpone this to a further work.

\section{Methodology}

The technique developed below for the detection of coronal loops consists of three steps: First we apply the 2D Morlet wavelet transform; it allows us to enhance the coronal loops over the background image. The next step is to segment the image thus obtained, in order to produce thinner loop traces and separate them from each other and from the background. Finally, we apply thresholding (Jiang, 2003; Soares et al., 2006), which eliminates false positives, that is, pixels that have been incorrectly labeled as belonging to loops. Since such pixels amount to noise, thresholding actually reduces the noise, so that the loops appear more clearly. In this way we keep only significant solar loops. 


\subsection{Application of the 2D Continuous Wavelet Transform to the Images of Coronal Loops}

The continuous wavelet transform (CWT) is the basic tool used in this work to detect curved structures, such as magnetic coronal loops. Let $s \in L^{2}\left(\mathbb{R}^{2}, \mathrm{~d}^{2} x\right)$ be an image (2D signal). Its two-dimensional continuous wavelet transform (with respect to the analyzing wavelet $\psi$ ) is simply defined as (Antoine et al., 2004):

$$
\begin{aligned}
S_{\psi}(\mathbf{b}, a, \theta) & =\left\langle\psi_{\mathbf{b}, a, \theta} \mid s\right\rangle \\
& =a^{-1} \int_{\mathbb{R}^{2}} \mathrm{~d}^{2} \mathbf{x} \psi^{*}\left(a^{-1} r_{-\theta}(\mathbf{x}-\mathbf{b})\right) s(\mathbf{x}) \\
& =a \int_{\mathbb{R}^{2}} \mathrm{~d}^{2} \mathbf{k} \mathrm{e}^{\mathrm{ib} \cdot \mathbf{k}} \widehat{\psi}^{*}\left(a r_{-\theta}(\mathbf{k})\right) \widehat{s}(\mathbf{k}),
\end{aligned}
$$

where $\widehat{\psi}$ denotes the Fourier transform of $\psi$, the asterisk denotes complex conjugation and $\mathbf{k}$ is the spatial frequency variable. Thus, the CWT is the projection of the signal $[s]$ on the (modified) analyzing wavelet $[\psi]$. The parameter $\mathbf{b} \in \mathbb{R}^{2}$ denotes a translation, $a>0$ a dilation, and $r_{-\theta}(0 \leq \theta<\pi)$ is the usual $2 \times 2$ rotation matrix. The properties of the wavelet imply that the CWT analysis is a local filtering in all four parameters $a, \theta$, and $\mathbf{b}=\left(b_{x}, b_{y}\right)$ : The CWT $S_{\psi}(\mathbf{b}, a, \theta)$ is appreciable only in those regions of parameter space where the transformed wavelet "matches" the signal. More details may be found in the appendix.

\subsubsection{Choice of an Adequate Analyzing Wavelet}

For the practical implementation, we have to choose a wavelet $[\psi]$. A variety of continuous wavelets are available for image processing (Antoine et al., 2004), and it is obvious that their shape should somehow reflect the signal to be extracted. In our study, we seek to detect coronal loops that are strongly elongated, oriented features. Therefore we have to choose a wavelet that is sensitive to direction. A wavelet which has a good capability to detect oriented features and is straightforward to implement is the 2D Morlet wavelet. The one we use (see (9) for a more general definition) is

$$
\psi_{M}(\mathbf{x})=\exp \left(\mathbf{i k}_{0} \cdot \mathbf{x}\right) \exp \left(-\frac{1}{2}|\mathbf{x}|^{2}\right)
$$

where the parameter $\mathbf{k}_{0}$ is the wave vector of the plane wave. Actually, other directional wavelets could also be used, such as the Cauchy or conical wavelets, but we expect that they would give essentially the same results. We choose the 2D Morlet wavelet for the purpose of this work, because it is easy to implement and well calibrated, and it offers the best trade-off between spatial and frequency resolutions (Antoine et al., 2004).

Following Soares et al. (2006), we reduce the four-dimensional parameter space $\{\mathbf{b}, a, \theta\}$ for $S_{\psi}$ by considering only the maximum of the modulus of the real part of the Morlet CWT over all possible orientations (for brevity we will speak simply of "the modulus" of the CWT), that is, over all values of the angle $[\theta]$,

$$
M_{\psi}(\mathbf{b}, a)=\max _{\theta}\left|\operatorname{Re} S_{\psi}(\mathbf{b}, a, \theta)\right| .
$$

The Morlet wavelet transform is calculated for various scales $[a]$ and angles $[\theta]$ varying from 0 to $170^{\circ}$ in steps of $10^{\circ}$. The maximum over all angles of the modulus of the real part 
of the CWT is taken as the pixel value for this scale $[a]$. Note that in (4) the maximum over the entire range $\theta \in[0, \pi]$ has to be searched in order to detect the loop orientation properly.

The angle $\theta_{\max }=\operatorname{argmax}\left|\operatorname{Re} S_{\psi}(\mathbf{b}, a, \theta)\right|$ at which the maximum is attained could also be recorded, but we do not use it here. For well-resolved loops, this angle, which should be aligned locally along the loop, is redundant, as the orientation of the loop is already given by the spatial distribution of $M_{\psi}$. In principle, the proper alignment of $\theta_{\max }$ could be used as a consistency check to exclude non-loop structures.

\subsubsection{Implementation}

For the numerical procedure, we must discretize the wavelet and evaluate it on the image pixel grid. The integral transform defining the CWT is most efficiently calculated in Fourier space as in (2). For a $N_{1} \times N_{2}$ image, this yields the following computation count: $N_{1} N_{2} \log \left(N_{1} N_{2}\right)$ operations for the forward FFT of an image, $N_{1} N_{2}$ for the multiplication with the wavelet FT, and $N_{1} N_{2} \log \left(N_{1} N_{2}\right)$ for the backward FFT of the product.

Since the image $[s]$ is discrete and has finite size, we have to choose the scale $a$ within an appropriate range, for which the CWT is meaningful (Antoine et al., 2002b; Jacques, 2004). This scale $[a]$ determines how well the wavelet $\left[\psi_{\mathbf{b}, a, \theta}\right]$ is sampled on the image grid and which scales it is capable to detect in the image. The support of the wavelet is proportional to $a$ in configuration space and proportional to $1 / a$ in Fourier space (see the full discussion in the appendix, in particular Figure 5). Obviously, the support should be well below the size of the discrete image in configuration and Fourier space, respectively. These two constraints define the allowed scale interval $\left[a_{\min }, a_{\max }\right]$. The Fourier size limitation leads to a value $a_{\min }$ considerably larger than the pixel size and ensures that the wavelet is not undersampled and does not "fall between" the image grid.

\subsubsection{Application of the Analyzing Wavelet to the Coronal Image}

We analyze the maximum of the 2D Morlet wavelet transform on a solar EUV image taken at $\lambda=171 \AA$ by the TRACE telescope (Handy et al., 1999). This line is emitted by Fe IX ions which are most abundant at about 1 million $\mathrm{K}$, the typical temperature of the lower corona. The image we use for testing our algorithm is of size $768 \times 768$, and was taken from an active region near the center of the solar disk on 14 July 1998 at 12:51:31 UT. It is extracted from the video sequence Trace-FLARE-JUL1498 and covers an area of width of $192000 \mathrm{~km}$. It is shown in Figure 1(a).

We tested for different values of the scale parameter $a$. For the smallest scale $a=5$ pixels, the loops in the maximum-modulus image appear thin and slightly separated from one another (Figure 1(b)). For increasing scales $a=7$ and 9 pixels, the loops become brighter and better separated (Figure 1(c) and (d)). At the same time, the noisy, low-level intensity variations in the background decrease with increasing scale.

Our goal is to determine the structure of coronal loops with a distinctly elongated shape. The wavelet that we use is designed to be sensitive to exactly this shape. The likelihood to pick up other bright objects that are not elongated like loops is highly reduced. For extended bright objects with a scale larger than $a$ like, e.g., moss on the solar surface, (1) gives little response because $\psi$ has a vanishing integral. Small bright features, like $e$.g. bright points or individual bright pixels enhanced by noise, yield a small contribution in (1) if their size is much smaller than the support of $\psi$. Also "negative" loops might occur, that is, bright lines, often straight, in the dark gap between close parallel bright loops. This is the case if one uses the modulus of the CWT itself in (4). An efficient way to eliminate these false 

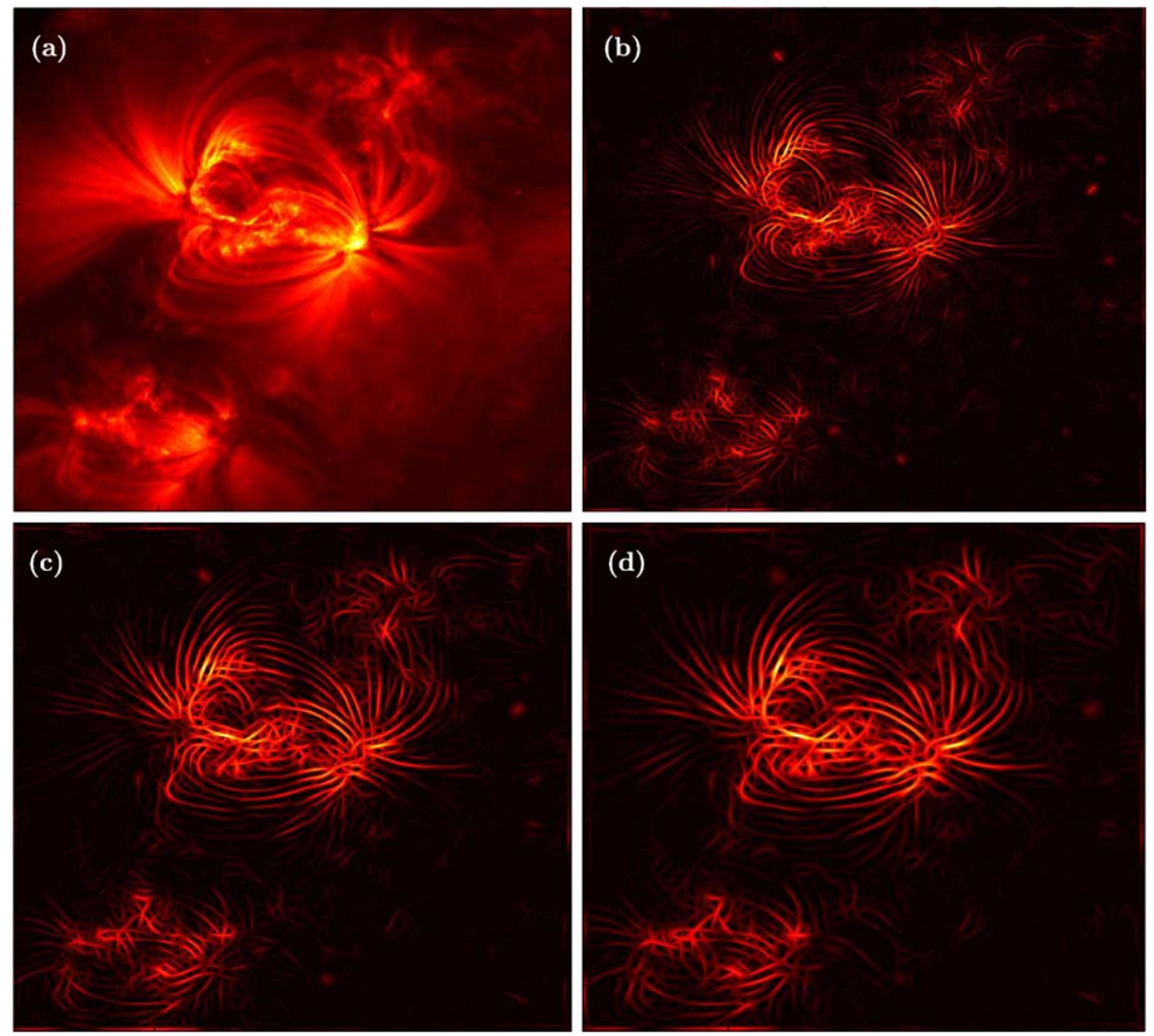

Figure 1 (a) Original image of the active region taken at $\lambda=171 \AA ̊$ by the TRACE telescope; (b) after using the maximum of the modulus of the real part of the Morlet WT for the scale $a=5$; (c) the same at scale $a=7$; and (d) the same at scale $a=9$. This image was taken at $\lambda=171 \AA$, on 14 July 1998 at 12:51:31 UT and covers an area $192000 \mathrm{~km}$ wide.

loops is to replace the modulus of the CWT by the modulus of its real part, as we have done throughout the work. This results in a clearer image, with clean, well-separated loops, and no "negative" loops.

In addition, some non-loop pixels are located differently at different scales, i.e. on the different images. Since genuine loop pixels do not depend on the scale, we treat these "unstable" pixels as noise. Therefore, the noise decreases for increasing scales, which precludes any superposition or scale averaging of several pictures at different scales, which would increase the noise considerably.

\subsection{Segmentation}

The purpose of image segmentation is to determine the outlines of different regions in the image; i.e., to divide the image into regions that are made up of pixels which have something in common. This is achieved by assigning a label to every pixel in the image, in such a way that pixels with the same label share certain visual characteristics and thus belong to the same region. For example, they may have similar brightness or intensity value, which may indicate that they belong to the same object. The resulting regions may indicate the presence 


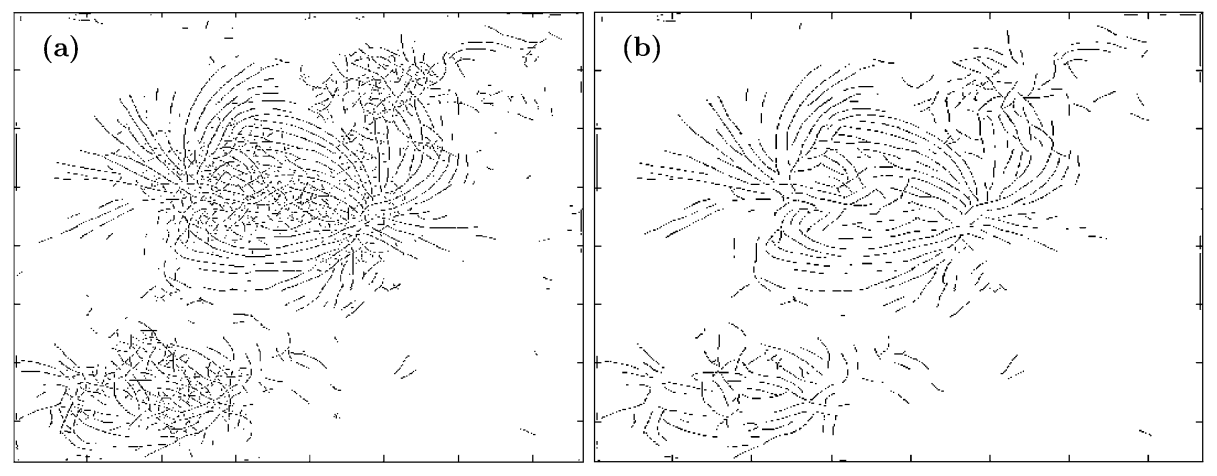

Figure 2 Segmentation of the TRACE image of Figure 1(c): (a) the noisy segmented image; (b) the same after the global and local thresholding.

of objects or parts of objects. Here, we segment the CWT modulus image by comparing pixels intensities with that of their nearest neighbors. As we have seen, the modulus of the real part of the wavelet transform is large for a pixel on a loop and low for background pixels. We label each pixel as a loop pixel if its modulus exceeds that of all its eight nearest neighbors. All other pixels, e.g. those that have a neighbor with a larger modulus value than the pixel itself are labeled as non-loop pixels. Of course, this procedure introduces discontinuities, since the resulting image consists of isolated pixels. Nevertheless, the algorithm allows us to follow closely the loop structure, despite the absence of the missing pixels (see Figure 2).

We show in Figure 2(a) the result of applying the segmentation to the image in Figure 1(c). Besides the obvious loop structures, the method occasionally selects several pixels from the noisy background as belonging to a loop structure if they are a local maximum. As we discussed above, objects which are not elongated as loops yield a small, but not completely vanishing, wavelet-transform modulus. Some of these small values happen to exceed all of their neighbor values and therefore contribute to the set of loop pixels. In a next step, we discriminate these "noise" pixels by their low modulus value.

\subsection{Thresholding}

Having segmented the image, we notice that its intelligibility is reduced by the presence of pixels of low intensity (most of which are falsely labeled as loop pixels) and, consequently, the loops are less visible. Of course, some of these pixels may belong to a loop, but also to other coronal phenomena. Anyway, since they do not yield complete information on the loop structure, we want to omit them and treat them as noise.

In order to reduce the noise induced by these pixels, we apply a combined global and local thresholding. With a global thresholding, all pixels of low intensity that are labeled as loop pixels are considered to be noise and are eliminated by a threshold $[T]$ determined from the histogram of the image. We present such a histogram in Figure 3, both for $a=7$ and $a=9$. In both cases, it is clear that the peaks on the left with small modulus intensities represent essentially noise and have to be eliminated. On the other hand, if we fix the threshold $[T]$ too high, we lose too much information, in particular, the loops become disconnected. Therefore, on an empirical basis, we fix the threshold for the intensity level at a modulus of $T=14$, which seems to give the best compromise in both cases. This value means that only the brightest $87 \%$ of the pixels are accepted. Thus, in order to improve image clarity 

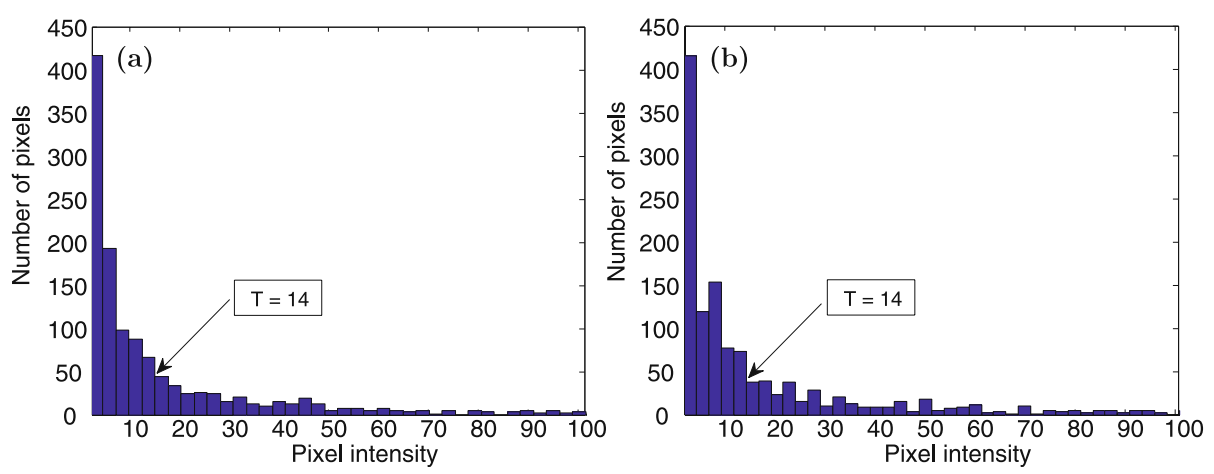

Figure 3 Histogram of the modulus $M_{\psi}(\mathbf{b}, a)$ of the transformed TRACE image: (a) for $a=7$; (b) for $a=9$. Empirically, the value $T=14$ of the threshold is the best choice.
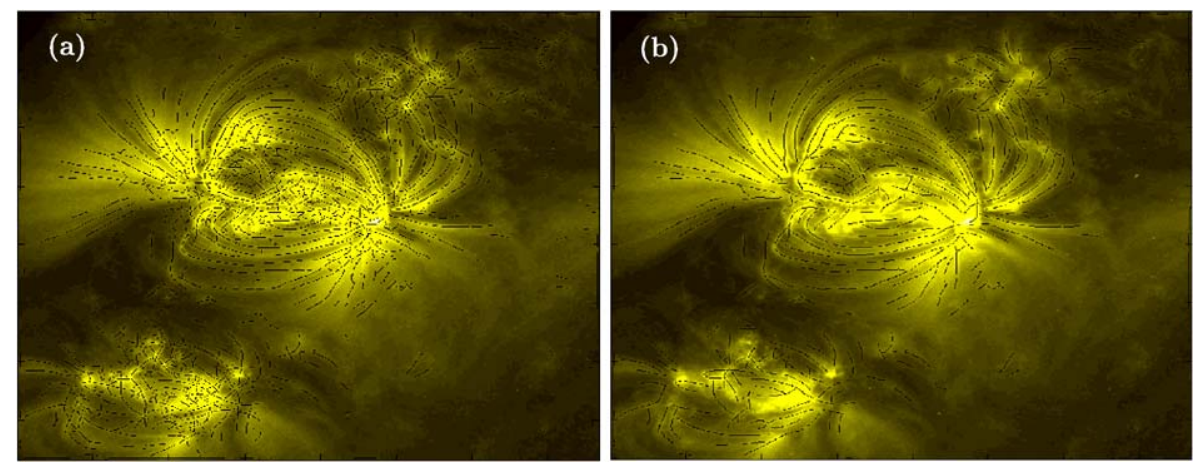

Figure 4 (a) Superposition of the original TRACE image shown in Figure 1(a) and the noisy segmented image from Figure 2(a); (b) the same after thresholding, Figure 2(b)

and highlight the coronal loops, we eliminate all pixels of intensity less than or equal to this threshold and the pixels whose intensity is greater than $T$ are considered as loop pixels.

The situation can be improved by applying an additional local thresholding to the previous result. This means that we divide the image into subregions and we compute an independent threshold for each subregion, namely the median intensity of the pixels within the subregion. A pixel is flagged as a background pixel (labeled non-loop pixel) if its intensity is lower than the local threshold, as a foreground pixel (labeled loop pixel) otherwise. In our case, we have divided the image into overlapping tiles of $15 \times 15$ pixels. For pixels that belong to the overlap between two tiles, the threshold is chosen as the mean of the two corresponding thresholds. Figure 2(b) shows the result after applying global and local thresholdings. This method gives reasonable results, since many, but not all, pixels falsely labeled as loop pixels in this image have been removed by the thresholdings.

As a final check, we have superposed in Figure 4 the results of Figure 2 on the original image. We see that the segmentation that we have applied indeed enhances the loops over the background: they have become more conspicuous. 


\section{Discussion and Conclusion}

We have presented an attempt to develop an automated segmentation method, called WTE, for loop structures in solar coronal images, by using the 2D Morlet continuous wavelet transform. The Morlet CWT is efficient at enhancing the contrast in loop structures, while filtering out noise.

The output of the WTE method is a subset of the image pixels labeled as loop pixels. This differs from other segmentation procedures that yield parameterized curve coordinates representing the loop axes. We have segmented the loops in a TRACE image by a wavelet transform. The initial set of loop pixels still contained some percentage of false pixels due to noise. These, however, could be successfully eliminated to a large extent by a combination of global and local thresholding.

Our technique produces reasonably good results: it has successfully highlighted loop structures on the TRACE telescope images, without losing an appreciable amount of pixelbased loop information. We notice that coronal-loop systems are often very complex; indeed, the magnetic-field lines show tangled network lines where loops do not appear clearly. This is why solar scientists call on 3D solar loop observations.

Our WTE scheme is robust and reasonably fast. For our $768 \times 768$ image, the result is obtained in about 25 seconds on a Intel Pentium $1.73 \mathrm{GHz}$ PC. The need for such schemes becomes pressing in view of the growing amount of solar EUV data that is accumulated by present and future solar missions. In particular, the AIA instrument of the SDO mission will produce EUV images at a cadence of approximately one image every ten seconds, which will lead to some 1.5 Terabytes of data per day. These images can no longer be inspected without support from fast and robust segmentation routines that may classify image objects and preselect data. Our present code cannot cope with such an image flow, so the next step is to accelerate it. This will be the subject of future work.

Coming back to the other methods described in the introduction, a direct comparison with similar procedures is difficult. An attempt has been made recently by Aschwanden et al. (2008a), but a genuine comparison is very difficult since all methods depend on various, noncomparable parameters. We emphasize that, in our method, only two parameters have to be fixed: the scale $[a]$ and the global threshold $[T]$. Most other methods have many more tuning parameters to be adjusted.

A legitimate question is the connection of the extracted loops with coronal magneticfield models, e.g. Wiegelmann (2008). For instance, one may wonder whether our results would give access to unobservable electric currents or to sub-resolution dynamics, but this leads far beyond the scope of the paper. The segmentation (or extraction) is just one step in a more complex analysis to constrain coronal-field models: it could provide the input for stereoscopy if applied to simultaneous image pairs, which would then allow a 3D reconstruction of the loops. Magnetic-field models should then reproduce this loop by one of its field lines. Alternatively, this field line should, if projected in the direction of the EUV observation, agree with the loop extraction. This check is not unambiguous, but still feasible if no stereoscopic image pair is available. How the field models are best modified to meet these constraints is beyond the scope of the paper.

Note that, if we would like to use the extracted loops for stereoscopy, it would be helpful if each individual loop could be identified and labeled. This requires an additional step to be applied to the loop pixels, which detects the shape of the connected loop pixel set, separates non-connected objects, and splits connected objects into overlapping loops. For other applications, e.g. loop brightness histograms or checking whether a model field line projects onto any extracted loop, such a separation would be unnecessary. This step is therefore deferred to a later investigation. 
Acknowledgements S. Biskri is supported by a doctoral fellowship from ADRI, Université catholique de Louvain. She gratefully acknowledges the hospitality and support of the Max-Planck-Institut for Solar System Research, Katlenburg-Lindau, Germany, as well as instructive discussions with J. de Patoul. She also thanks V. Delouille (Observatoire Royal de Belgique, Brussels, Belgium) for providing the image shown in Figure 1(a). Finally, the authors thank the referees for their constructive suggestions, which have much improved the paper.

Open Access This article is distributed under the terms of the Creative Commons Attribution Noncommercial License which permits any noncommercial use, distribution, and reproduction in any medium, provided the original author(s) and source are credited.

\section{Appendix: The 2D Continuous Wavelet Transform}

The 1D continuous wavelet transform is well-known and all the necessary information can be found in several textbooks, for instance of Mallat (1999) or Torrésani (1995). In two dimensions, however, the literature is very scarce, and the only systematic treatment is the monograph by one of us (Antoine et al., 2004). Hence we will recall here the bare essentials needed to understand our method.

Let $s \in L^{2}\left(\mathbb{R}^{2}, \mathrm{~d}^{2} \mathbf{x}\right)$ be an image, that is a 2D signal of finite energy. Its two-dimensional continuous wavelet transform, with respect to the fixed wavelet $\psi$, is defined as the scalar product of $s$ with $\psi$, in the sense of $L^{2}\left(\mathbb{R}^{2}, \mathrm{~d}^{2} \mathbf{x}\right)$ :

$$
\begin{aligned}
S_{\psi}(\mathbf{b}, a, \theta) & =\left\langle\psi_{\mathbf{b}, a, \theta} \mid s\right\rangle \\
& =a^{-1} \int \mathrm{d}^{2} \mathbf{x} \psi^{*}\left(a^{-1} r_{-\theta}(\mathbf{x}-\mathbf{b})\right) s(\mathbf{x}), \\
& =a \int_{\mathbb{R}^{2}} \mathrm{~d}^{2} \mathbf{k} \mathrm{e}^{\mathrm{ib.k}} \widehat{\psi}^{*}\left(a r_{-\theta}(\mathbf{k})\right) \widehat{s}(\mathbf{k}),
\end{aligned}
$$

where the asterisk denotes complex conjugation, $\psi_{\mathbf{b}, a, \theta}$ is a copy of $\psi$ translated by $\mathbf{b} \in \mathbb{R}^{2}$, dilated by a factor $a>0$, and rotated by an angle $\theta \in[0,2 \pi]$, that is,

$$
\psi_{\mathbf{b}, a, \theta}=\frac{1}{a} \psi\left(\frac{1}{a} r_{\theta}^{-1}(\mathbf{x}-\mathbf{b})\right)
$$

where $r_{\theta}$ is the familiar $2 \times 2$ rotation matrix of angle [ $\left.\theta\right]$. The relation (6) shows that the CWT may be interpreted as a (continuous) filterbank. This also means that additional geometric restrictions on the wavelet (support properties, for instance) must be imposed on $\widehat{\psi}$, not on $\psi$.

In order to have an acceptable transform, the wavelet $[\psi]$ must satisfy an admissibility condition which, for all practical purposes, may be taken as a zero mean condition:

$$
\widehat{\psi}(\mathbf{0})=0 \Longleftrightarrow \int \mathrm{d}^{2} \mathbf{x} \psi(\mathbf{x})=0 .
$$

Since the wavelet $[\psi]$ is required to have zero mean, the CWT provides a filtering effect, i.e., the analysis is local in all four parameters $\mathbf{b}, a, \theta$. In other words, the CWT selects the component of $s$, if any, that "lives" at the location $\mathbf{b}$, at the scale $a$ and in the direction $\theta$. A straightforward calculation yields a Plancherel relation for the CWT, namely, $\|s\|^{2}=\left\|S_{\psi}\right\|^{2}$, which manifests the fact that no energy is lost in the CWT parameter space (indeed, this relation implies that $|S|^{2}$ can be interpreted as an energy density in the CWT). As a further 
$(a)$

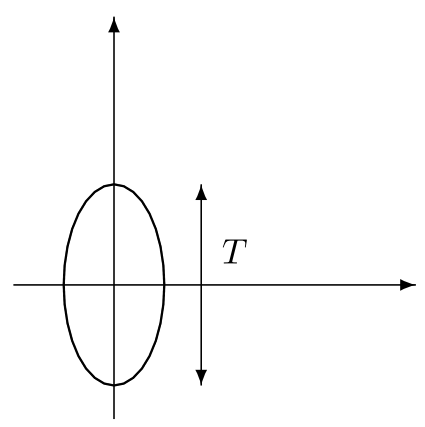

(c)

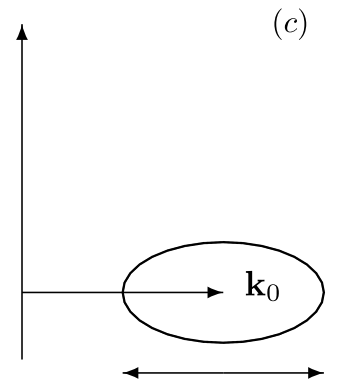

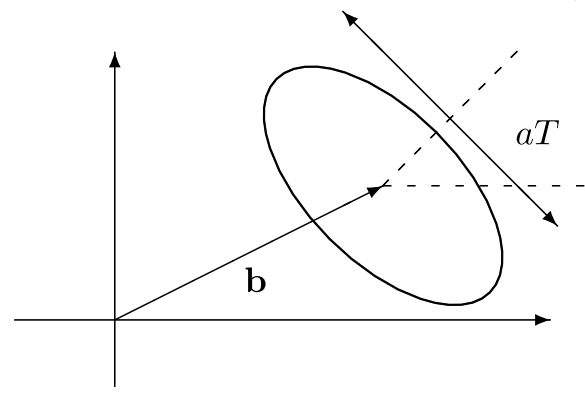

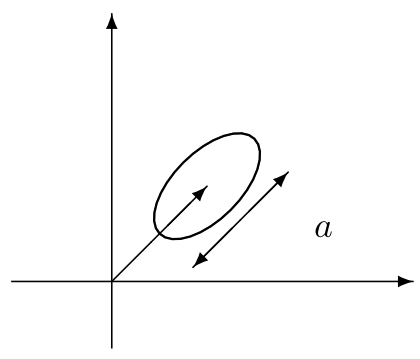

(d)

Figure 5 Support properties under the basic operations: (top) in the time domain: (a) the original signal $\psi(\mathbf{x})$; (b) the modified signal $\psi_{\mathbf{b}, a, \theta}(\mathbf{x})$, with $\mathbf{b}=(2.4,1.2), a=1.5, \theta=45^{\circ}$; (bottom) in the frequency domain: (c) the original signal $\widehat{\psi}(\mathbf{k}) ;$ (d) the modified signal $\widehat{\psi_{\mathbf{b}, a, \theta}}(\mathbf{k})$.

consequence, one obtains an exact reconstruction formula of the image from its wavelet transform.

The efficiency of the CWT at detecting singularities in images rests upon the support properties of the analyzing wavelet. First, we have to assume that the wavelet function $[\psi]$ and its Fourier transform $[\widehat{\psi}]$ are both well localized, in position $[\mathbf{x}]$ and in spatial frequency [k], respectively, which means that they are numerically negligible outside of bounded subsets of $\mathbb{R}^{2}$ in both variables $\mathbf{x}$ and $\mathbf{k}$ (they cannot vanish outside of both subsets, because of Fourier's uncertainty theorem). More precisely, assume $\psi$ has for numerical support a "disk" of diameter $T$, centered around $\mathbf{0}$, while $\widehat{\psi}$ has for numerical support a "disk" of diameter $\Omega$, centered around $\mathbf{k}_{0}$. Then, for the transformed wavelets $\psi_{\mathbf{b}, a, \theta}$ and $\widehat{\psi_{\mathbf{b}, a, \theta}}$, we have, respectively:

- the numerical support of $\psi_{\mathbf{b}, a, \theta}$ is a "disk" of diameter $a T$ centered around $\mathbf{b}$ and rotated by an angle $\theta$;

- the numerical support of $\widehat{\psi_{\mathbf{b}, a, \theta}}$ is a "disk" of diameter $\Omega / a$, centered around $r_{\theta}\left(\mathbf{k}_{0}\right) / a$ and rotated by $\theta$.

This property is illustrated in Figure 5. Note that the product of the two diameters is constant (it has to be bounded below by a fixed constant, by Fourier's theorem). As a consequence, we may characterize the filter properties of the wavelet. 
- If $a \gg 1, \psi_{\mathbf{b}, a, \theta}$ is a wide window, whereas $\widehat{\psi_{\mathbf{b}}, a, \theta}$ is very peaked around a small spatial frequency $r_{\theta}\left(\mathbf{k}_{0}\right) / a$ : this transform will be most sensitive to low spatial frequencies.

- If $a \ll 1, \psi_{\mathbf{b}, a, \theta}$ is a narrow window and $\widehat{\psi_{\mathbf{b}, a, \theta}}$ is wide and centered around a high spatial frequency $r_{\theta}\left(\mathbf{k}_{0}\right) / a$ : this wavelet has a good localization capability in the space domain and is mostly sensitive to high spatial frequencies.

Thus wavelet analysis operates at constant relative bandwidth $[\Delta k / k=$ const] where $k \equiv$ $\|\mathbf{k}\|$. Therefore, the analysis is most efficient at high spatial frequencies or small scales, and so it is particularly apt at detecting discontinuities in images, either point or line singularities (contours, corners) or directional features (edges, segments). In other words, the CWT is a singularity scanner. Clearly the coronal loops are of this type, which justifies the use of the CWT for extracting them.

The next step is to choose an analyzing wavelet $[\psi]$. At this point, there are two possibilities, depending on the problem at hand: If one chooses a wavelet invariant under rotation, the orientation parameter $[\theta]$ drops out and one performs a pointwise analysis, without direction selectivity. On the contrary, a directional wavelet is good for detecting oriented features. Features present in solar coronal-loop images taken by TRACE, such as the curved shape of the loops, suggest using a directional wavelet, for that reason. Thus, for the purpose of this work, we have chosen a typical directional wavelet, namely, the isotropic 2D Morlet wavelet, which is defined as:

$$
\psi_{\mathrm{M}}(\boldsymbol{x})=\exp \left(\mathbf{i k}_{0} \cdot \mathbf{x}\right) \exp \left(-\frac{1}{2}|\mathbf{x}|^{2}\right)+c(\mathbf{x}),
$$

where the parameter $\mathbf{k}_{0}$ is the wave vector. The correction term [c] must be added in order to satisfy the admissibility condition (8), but it is numerically negligible for $\left|\mathbf{k}_{0}\right| \geq 5.6$, so that it can be ignored in practice.

Of course, other choices are possible, such as an anisotropic Morlet wavelet (the Gaussian in the exponent is elongated in one direction) or a conical wavelet (whose Fourier transform $[\widehat{\psi}]$ vanishes identically outside a convex cone with apex at the origin in $\mathbf{k}$-space). The present choice is the simplest one and the isotropic 2D Morlet wavelet is well calibrated. In addition, because of its (essentially) Gaussian character, it offers the best balance between space and frequency localization. For the other wavelets mentioned here, we refer to the monograph of Antoine et al. (2004).

\section{References}

Antoine, J.P., Carrette, P., Murenzi, R., Piette, B.: 1993, Image analysis with two-dimensional continuous wavelet transform. Signal Process. 31, 241 - 272.

Antoine, J.P., Demanet, L., Jacques, L., Hochedez, J.F., Terrier, R., Verwichte, E.: 2002a, Application of the 2-D wavelet to astrophysical images. Phys. Mag. 24, $93-116$.

Antoine, J.P., Demanet, L., Jacques, L., Vandergheynst, P.: 2002b, Wavelets on the sphere: Implementation and approximations. Appl. Comput. Harmon. Anal. 13, 177-200.

Antoine, J.P., Murenzi, R., Vandergheynst, P., Ali, S.T.: 2004, Two-dimensional Wavelets and Their Relatives, Cambridge University Press, Cambridge. Chapter 1.

Aschwanden, M.J., Nightingale, R.W., Alexander, D.: 2000, Evidence for nonuniform heating of coronal loops inferred from multithread modeling of trace data. Astrophys. J. 541, 1059-1077.

Aschwanden, M.J., Lee, J.K., Gary, G.A., Smith, M., Inhester, B.: 2008a, Comparison of five numerical codes for automated tracing of coronal loops. Solar Phys. 248, 359-377. doi:10.1007/s11207-007-9064-9.

Aschwanden, M.J., Nitta, N.V., Wülser, J.P., Lemen, J.R.: 2008b, First 3D reconstructions of coronal loops with the STEREO A + B spacecraft. II. Electron density and temperature measurements. Astrophys. J. 680, 1477 - 1495. doi:10.1086/588014. 
Carcedo, L., Brown, D.S., Hood, A.W., Neukirch, T., Wiegelmann, T.: 2003, A quantitative method to optimize magnetic field line fitting of observed coronal loops. Solar Phys. 218, 29-40.

Delouille, V., de Patoul, J., Hochedez, J.F., Jacques, L., Antoine, J.P.: 2005, Wavelet spectrum analysis of EIT/SoHO images. Solar Phys. 228, $301-321$.

Farge, M., Kevlahan, N.K.R., Perrier, V., Schneider, K.: 1998, Turbulence analysis, modelling and computing using wavelets. In: van den Berg, J. (ed.) Wavelets in Physics, Cambridge Univ. Press, Cambridge, 117 200 .

Feng, L., Inhester, B., Solanki, S.K., Wiegelmann, T., Podlipnik, B., Howard, R.A., Wülser, J.P.: 2007, First stereoscopic coronal loop reconstructions from STEREO-SECCHI images. Astrophys. J. 671, 205-208. doi:10.1086/525525.

Gary, G.A.: 2001, Plasma beta above a solar active region: Rethinking the paradigm. Solar Phys. 203, 71 86.

Handy, B.N., Acton, L.W., Kankelborg, C.C., Wolfson, C.J., Akin, D.J., Bruner, M.E., Caravalho, R., Catura, R.C., Chevalier, R., Duncan, D.W., Edwards, C.G., Feinstein, C.N., Freeland, S.L., Friedlaender, F.M., Hoffmann, C.H., Hurlburt, N.E., Jurcevich, B.K., Katz, N.L., Kelly, G.A., Lemen, J.R., Levay, M., Lindgren, R.W., Mathur, D.P., Meyer, S.B., Morrison, S.J., Morrison, M.D., Nightingale, R.W., Pope, T.P., Rehse, R.A., Schrijver, C.J., Shine, R.A., Shing, L., Strong, K.T., Tarbell, T.D., Title, A.M., Torgerson, D.D., Golub, L., Bookbinder, J.A., Caldwell, D., Cheimets, P.N., Davis, W.N., DeLuca, E.E., McMullen, R.A., Warren, H.P., Amato, D., Fisher, R., Maldonado, H., Parkinson, C.: 1999, The transition region and coronal explorer. Solar Phys. 187, 229-260. doi:10.1023/A:1005166902804.

Inhester, B., Feng, L., Wiegelmann, T.: 2008, Segmentation of loops from coronal EUV images. Solar Phys. 248, $379-393$.

Jacques, L.: 2004, Ondelettes, repères et couronne solaire. PhD thesis, Université Catholique de Louvain, Louvain-la-Neuve.

Jiang, X.: 2003, Adaptive local threshold by verification-based multithreshold probing with application to vessel detection in retinal images. IEEE Trans. Pattern Anal. Mach. Intell. 25, 131 - 137.

Lee, J.K., Newman, T.S., Gary, G.A.: 2006a, Dynamic aperture-based solar loop segmentation. In: SSIAI '06: Proceedings of the 2006 IEEE Southwest Symposium on Image Analysis and Interpretation, IEEE Computer Society, Washington, 91 -94. ISBN 1-4244-0069-4. doi:10.1109/SSIAI.2006.1633725.

Lee, J.K., Newman, T.S., Gary, G.A.: 2006b, Oriented connectivity-based method for segmenting solar loops. Pattern Recogn. 39, 246-259.

Lindeberg, T.: 1998, Edge detection and ridge detection with automatic scale. Int. J. Comput. Vis. 30(2), $117-154$.

Mallat, S.G.: 1999, A Wavelet Tour of Signal Processing, Academic Press, San Diego.

McEwen, J.D., Vielva, P., Wiaux, Y., Barreiro, R.B., Cayon, L., Hobson, M.P., Lasenby, A.N., MartinezGonzalez, E.: 2006, Cosmological applications of a wavelet analysis on the sphere. J. Fourier Anal. Appl. 13, $495-510$.

Sakai, J.I., Furusawa, K.: 2002, Nonuniform heating of coronal loop footpoints and formation of loop threads associated with up- and downflows in the solar chromosphere. Astrophys. J. 564, 1048-1053. doi:10.1086/324133.

Slezak, E., Bijaoui, A., Mars, G.: 1990, Identification of structures from galaxy counts. Use of the wavelet transform. Astron. Astrophys. 227, $301-316$.

Soares, J.V.B., Leandro, J.J.G., Cesar, J.R.-M., Jelinek, H.F., Cree, M.J.: 2006, Retinal vessel segmentation using the 2-D Morlet wavelet and supervised classification. IEEE Trans. Med. Imag. 25, 1214-1222.

Strous, L.: 2002, Loop detection, http://www.Imsal.com/ aschwand/stereo/2000_easton/cdaw.html.

Torrésani, B.: 1995, Analyse continue par ondelettes. InterEditions, CNRS Editions, Paris.

Wiegelmann, T.: 2008, Nonlinear force-free modeling of the solar coronal magnetic field. J. Geophys. Res. 113(A12), 3. doi:10.1029/2007JA012432. 